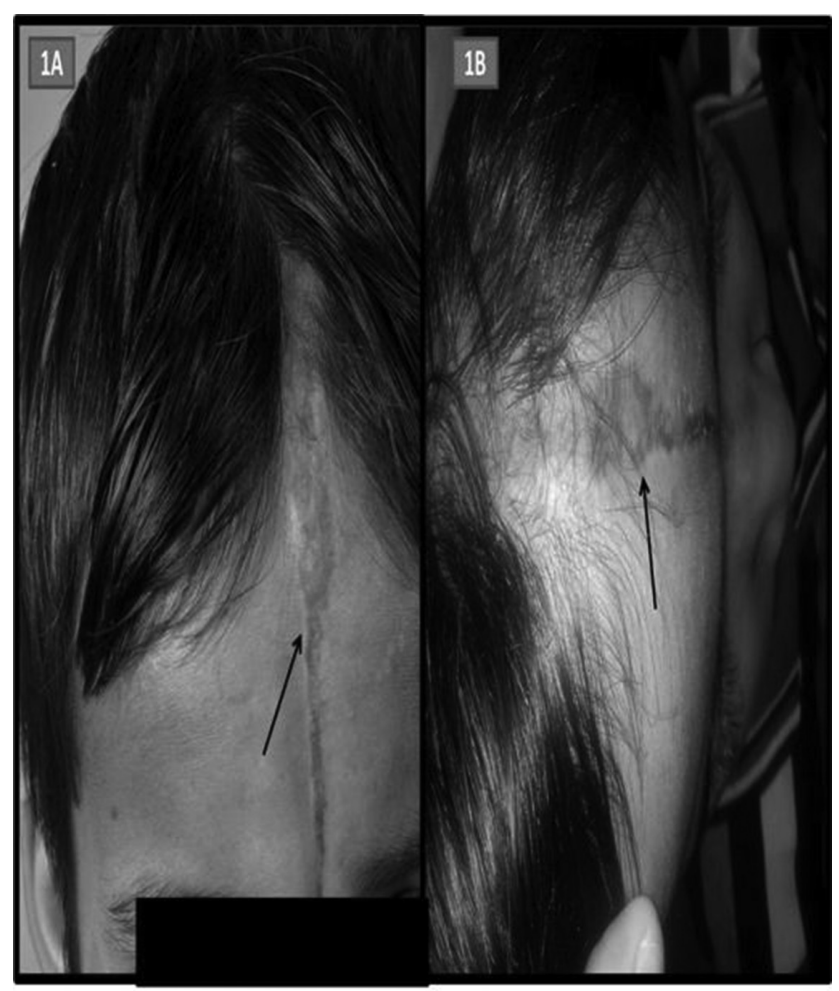

Abstract 368 Figure 1

and $\mathrm{V}$ sign. She also had swallowing difficulty proven by abnormal videofluoroscopic swallowing test. Laboratory findings showed anaemia, thrombocytopenia, elevated muscle enzymes, hyperferritinemia and hypertriglyceridemia. The autoimmune profile revealed positive antinuclear antibody (1:160, homogenous pattern) and negative anti-Jo-1 antibody. In addition to electromyography and skeletal muscle biopsy, bone marrow biopsy was performed to find the cause of microangiopathic hemolytic anaemia and thrombocytopenia. Numerous CD68-positive macrophages engulfing erythrocytes and platelets were revealed in bone marrow study. She was finally diagnosed as DM with secondary HPS. After steroid pulse therapy for 3 days, we continued high dose steroid therapy for 1 month. Thereafter, we gradually tapered the steroid and started methotrexate. After 1 year of treatment, she was completely recovered from muscle weakness, swallowing difficulty, skin lesions and cytopenia.

Conclusions With this unique case, we would like to assert that HPS should be considered when cytopenia is observed in the patients with DM and that early aggressive therapy is needed.

\section{THE CLINICAL CHARACTERISTICS OF SARCOID ARTHROPATHY BASED ON A PROSPECTIVE COHORT STUDY}

${ }^{1} \mathrm{~S}$ Kobak*, ${ }^{2} \mathrm{~F}$ Sever, ${ }^{3} \mathrm{O}$ Usluer, ${ }^{4} \mathrm{~T}$ Goksel, ${ }^{5} \mathrm{M}$ Orman. ${ }^{1}$ Istinye University Faculty of Medicine -LIV Hospital, Rheumatology, Istanbul, Turkey; ${ }^{2}$ Medicalpark Hospital, Chest Diseases, Izmir, Turkey; ${ }^{3}$ Suat Seren Chest Diseases Hospital, Chest Surgery, Izmir, Turkey; ${ }^{4}$ Ege University Faculty of Medicine, Chest Diseases, Izmir, Turkey; ${ }^{5}$ Ege University Faculty of Medicine, Statistics, Izmir, Turkey

10.1136/lupus-2017-000215.370
Background and aims Sarcoidosis is known as a Th1-mediated disease which can mimic many primary rheumatologic diseases or sometimes co-exist with them. Clinical characteristics of sarcoid arthropathy are not well described and the studies reported in the literature so far are mostly based on the data from referrals. The aim of this study was to evaluate the incidence and clinical characteristics of sarcoid arthropathy.

Methods All our patients were prospective evaluated in our single Rheumatology outpatient centre from 2011 to 2015. 114 patients with sarcoidosis were included in the study.

Results The mean patient age was 48.1 years and the mean disease duration was 40.5 months.Sarcoid arthritis was observed in 71 (62.3\%), and arthralgia in 106 (92.9\%) patients. Out of the 71 patients with arthritis, $61(85.9 \%)$ had involvement of ankle, 7 (9.8\%) knee, 2 (2.8\%) wrist, MCP and PIP joints, and one (1.4\%) had shoulder periarthritis. Oligoarthritis (two to four joints) was the most common pattern followed by monoarthritis and polyarthritis. When the correlation between clinical findings was considered, erythema nodosum and arthritis and female gender were found to be correlated $(p=0.03, p=0.001$, respectively). Again in patients with arthritis, even higher levels of CRP/ESR as well as ANA and RF positivity were observed $(p=0.03, p=0.01, p=0.01$ and $p=0.02$, respectively). Eleven patients had another rheumatic pathology concurrent with sarcoidosis.

Conclusions Inflammatory arthritis occurs in a majority of patients with sarcoidosis. Acute arthritis with bilateral ankle involvement is the most common pattern of sarcoid arthropathy. Sarcoidosis can mimic many primary rheumatic diseases and/or may coexist with them.

\section{THE USE OF HAND PERFUSION SCINTIGRAPHY TO ASSESS RAYNAUD'S PHENOMENON ASSOCIATED WITH HAND-ARM VIBRATION SYNDROME}

${ }^{1}$ SH Lee ${ }^{*},{ }^{1} \mathrm{KA}$ Lee, ${ }^{1} \mathrm{HR}$ Kim, ${ }^{2} \mathrm{HW}$ Chung. ${ }^{1}$ Konkuk University Medical Centre, Rheumatology, Seoul, Republic of Korea; ${ }^{2}$ Konkuk University Medical Centre, Nuclear medicine, Seoul, Republic of Korea

\subsection{6/lupus-2017-000215.371}

Background and aims This study aimed to evaluate the hand perfusion scintigraphic features of hand-arm vibration syndrome (HAVS) and to compare these with the features of primary and secondary Raynaud's phenomenon (RP) associated with rheumatic diseases.

Methods Hand perfusion scintigraphy was performed in 57 patients with primary RP, 71 patients with HAVS-related RP, and 36 patients with rheumatic disease-related RP. Patients' clinical details were collected by a retrospective review of medical records. We calculated 6 ratios by using the timeactivity curve and static blood pool images, the chilled to ambient hand and wrist ratios of the first peak height, initial slope, and blood pool uptake. We analysed 3 morphologic characteristics: slow progress pattern, paradoxically increased uptake pattern in the time-activity curve, and the inhomogeneous radioactivity uptake in the blood pool image.

Results All of the 71 patients with HAVS-related RP were mine workers. The onset of RP after exposure to vibration was at $21.8 \pm 7.3$ years, with $26.3 \pm 7.0$ years of vibration exposure time. The chilled to ambient hand ratios of the first peak height and the initial slope were significantly lower in patients with HAVS-related occupational RP than in patients with primary RP. The presence of a paradoxically increased 
uptake pattern was significantly lower in HAVS than in primary RP.

Conclusions There were significant differences in hand perfusion scintigraphic features between primary RP and HAVS. These results suggest that the underlying pathophysiology of the two diseases differs; thus, different criteria should be applied for their evaluation.

\section{CLINICAL FEATURES, OUTCOMES AND RISK FACTORS FOR THE DEVELOPMENT OF POSTERIOR REVERSIBLE ENCEPHALOPATHY SYNDROME (PRES) IN THAI PATIENTS WITH SYSTEMIC LUPUS ERYTHEMATOSUS} (SLE)

${ }^{1} \mathrm{U}$ Damrongpipatkul, ${ }^{1} \mathrm{~N}$ Kasitanon, ${ }^{2} \mathrm{~K}$ Oranratanachai, ${ }^{1} \mathrm{~W}$ Louthrenoo*. ${ }^{1}$ Faculty of Medicine Chiang Mai University, Division of Rheumatology Department of Internal Medicine, Chiang Mai, Thailand; ${ }^{2}$ Faculty of Medicine Chiang Mai University, Division of Neuroimaging and Interventional Radiology Department of Radiology, Chiang Mai, Thailand

\subsection{6/lupus-2017-000215.372}

Background and aims PRES in SLE is increasingly recognised. This study aimed at determining the prevalence, clinical features, brain imaging findings, risk factors, and outcomes of PRES in Thai SLE patients.

Methods SLE patients with PRES seen between 1 January 1986 and 31 August 2013 were identified. Controls were matched with hospital number and disease duration to cases (ratio, 1:4). Clinical features, brain imaging patterns, risk factors, treatment and outcome of PRES were determined.

Results Of 1141 SLE patients, 26 PRES episodes occurred 20 in females (prevalence 1.8\%). Mean $\pm \mathrm{SD}$ age at diagnosis and disease duration was $29.3 \pm 13.1$ and $2.8 \pm 3.4$ years, respectively. Among the 26 episodes, 24 (92.3\%) had seizure, 14 $(53.8 \%)$ headache, $9(34.6 \%)$ fever and vomiting and 8 (30.8\%) visual disturbance. All of them had acutely elevated blood pressure. 20 and 23 patients had active lupus nephritis (LN) within 3 months prior to and at PRES onset, respectively. Dominant parietal-occipital pattern was the most common brain imaging abnormality. 22 episodes improved with blood pressure control. Immunosuppressive therapy was given for active disease in 8 episodes. Anti-convulsive therapy could be discontinued in 21 of 22 episodes (median duration 3 months). Auto-immune hemolytic anaemia (AIHA) and LN were PRES risk factors (OR 6.55, 95\% CI 1.09-39.39, $\mathrm{p}=0.04$ and $\mathrm{OR} 3.06,95 \%$ CI 1.12-8.39, $\mathrm{p}=0.03$, respectively). 6 patients $(23.1 \%)$ died during PRES episodes. The mortality rate in SLE patients with PRES was significantly higher than those without $(30 \%$ vs. $10 \%, \mathrm{p}<0.001)$.

Conclusions The mortality rate was high in Thai SLE with PRES. AIHA and LN were risk factors for PRES.

\section{ASSOCIATION BETWEEN DISEASE ACTIVITY, QUALITY OF LIFE AND QUALITY OF SLEEP IN PATIENTS WITH SYSTEMIC LUPUS ERYTHEMATOSUS (SLE)}

'D Monova, ${ }^{2} S$ Monov*. 'Medical University - Sofia- Medical Institute, Department of Internal Diseases, Sofia, Bulgaria; ${ }^{2}$ Medical University - Sofia, Department of Internal Diseases- Clinic of Rheumatology, Sofia, Bulgaria

10.1136/lupus-2017-000215.373
Background and aims The aim of this study was to examine the association of SLE disease activity, quality of life and quality of sleep in patients diagnosed with SLE.

Methods 132 SLE patients with a confirmed diagnosis of SLE, according ACR classification criteria, were enrolled in this study. The patients completed the following questionnaires: the Pittsburg Sleep Quality Index (PSQI), the 12 item Short Form Health survey (SF-12), the Lupus Patient-Reported Outcome tool (PupusPRO), SLE Quality of Life Questionnaire (LQoL). Clinical information, including the SLE Disease Activity Index (SLEDAI), was obtained from medical records. Student's t-test, ANOVA, Pearson correlation measured were used in statistical analysis.

Results The majority of the participants $(84,4 \%)$ had sleep disturbances (PSQI >5). Total PSQI score was weakly associated with all of the SF-12 subcategories and showed weak to moderate associations with LupusPRO subcategories $(\mathrm{r}<0,05)$, except for medication $(r<0,2)$. "Sleep efficiency" was weakly associated with "physical health", "physical function", and "paim" in the SF-12 and LupusPRO. "Sleep quality" and "sleep disturbances" were weakly associated with "pain" and the "emotional" and "mental" subcategories in the SF-12 and LupusPRO. SLE QoL was significantly higher in patients with good sleep.

Conclusions We found that quality of sleep, especially "sleep efficiency", was poor for the majority of patients with SLE. Quality of sleep was associated with various aspects of quality of life, especially pain, vitality, and emotional health. Management of pain and emotional health may be important for improving quality of sleep in SLE patients.

\section{ANA NEGATIVE RENAL LIMITED LUPUS NEPHRITIS -A RARE ENTITY}

${ }^{1} \mathrm{SP}$ Nagaraju*, ${ }^{1} \mathrm{RP}$ Attur, ${ }^{1} \mathrm{D}$ Rangaswamy, ${ }^{2} \mathrm{SL}$ Koulmane Laxminarayana, ${ }^{1} \mathrm{SP}$ Rao, ${ }^{1} \mathrm{~S}$ Kaza, ${ }^{1} \mathrm{~K}$ Saraf, 'S Shenoy, 'M Bhojaraja, ${ }^{1} \mathrm{~A}$ Rangaswamy, ${ }^{3} \mathrm{~V}$ Mahesha. ${ }^{1}$ Kasturba Medical college- Manipal University, Nephrology, Manipal, India; ${ }^{2}$ Kasturba Medical college- Manipal University, pathology, Manipal, India; ${ }^{3}$ Manipal HospitalBangalore, Pathology, Bangalore, India

\subsection{6/lupus-2017-000215.374}

Background and aims Antinuclear antibodies (ANA) in serum is considered a decisive diagnostic test for SLE. ANA negative SLE is a subgroup of SLE that is infrequently recognised. We report an unusual case of seronegative SLE which presented

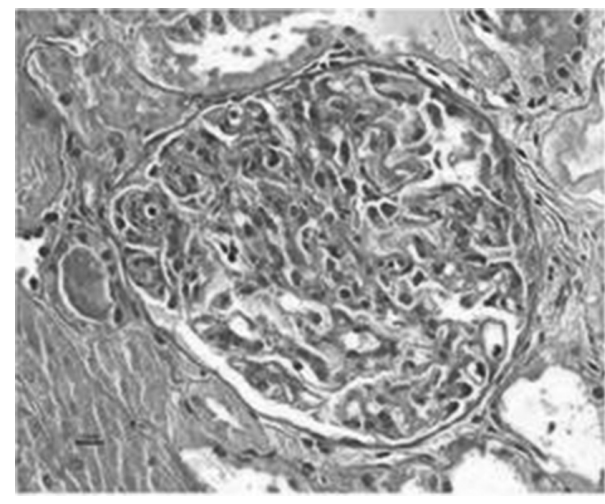

Abstract 374 Figure 1 